\title{
Hypertension and glomerular diseases: the importance of a holistic approach
}

\author{
Paolo Cravedi ${ }^{1}$. Jeremy S. Leventhal ${ }^{2}$. Giorgina B. Piccoli ${ }^{3,4}$
}

Published online: 12 February 2021

(c) Italian Society of Nephrology 2021

In this issue of the journal, the Korean GlomeruloNEphritis Study (KoGNET) Group reports on the impact of bloodpressure levels and disease activity on renal outcomes in a cohort of 1066 patients with glomerulonephritis [1]. Both control of hypertension and remission status were independently associated with $\mathrm{a} \geq 50 \%$ lower risk of a decrease in estimated glomerular filtration rate (eGFR) and end-stage kidney disease (ESKD). Associations were more prominent in patients with higher-grade proteinuria. This finding is consistent with a large body of literature showing that blood-pressure control [2], as well as life-style interventions, including promoting healthy dietary habits, and regular physical activity, can slow the progression of chronic kidney disease (CKD) [3].

Interestingly, the Korean study shows that over time blood-pressure control, rather than levels at diagnosis of glomerulonephritis is more closely correlated with the risk of CKD progression [REF JN paper]. Although renin-angiotensin-aldosterone system (RAAS) inhibitors were additionally beneficial in patients with proteinuria, better bloodpressure control benefited patients independently of the anti-hypertensive agent used [REF JN paper]. Therefore, the retrospective data suggest that lowering blood pressure is an actionable therapeutic target and should be prioritized in patients with glomerular diseases, once remission of disease has been achieved.

Paolo Cravedi

paolo.cravedi@mssm.edu

1 Department of Medicine, Icahn School of Medicine at Mount Sinai, Translational Transplant Research Center, New York, NY, USA

2 Division of Nephrology, White Plains Hospital, White Plains, NY, USA

3 Centre Hospitalier Le Mans, Le Mans, France

4 Department of Clinical and Biological Sciences, University of Torino, Torino, Italy
A vast armamentarium of anti-hypertensive agents provides myriad solutions for hypertension control. Aided by widely available personal ambulatory measurement devices and 24-h monitoring, most patients should be able to receive personalized targeted treatment [4]. Proportionally small risks and costs of anti-hypertensives compared to immunosuppressive therapies further support renewed focus on their role in glomerulonephritis management. While multipledrug therapy is associated with a high risk of non-compliance, patients with glomerulonephritis may find additional motivation for adherence if they have a better understanding of the risks associated with ESKD and realize that by failing to take medication they could become increasingly ill.

Given the proven benefits of targeted blood-pressure control, failure to attain blood- pressure goals in patients with glomerulonephritis is surprising. Perhaps the lack of convincing data on the effect of blood-pressure control on CKD progression, specifically in patients with glomerulonephritis, has contributed to a minimalist approach.

How can we do better? Decades of treating chronic diseases, such as diabetes, and caring for patients with organ transplantation suggest that integrated specialist-based care is a superior management policy [5-7]. Counterintuitively, recent randomized and observational studies challenge this view, contending that guideline-driven care yields at least the same results as multidisciplinary, nephrology-driven care [8].

The issue is probably more complex than it seems. The devil, as well as the angel, is in the details. Differences between a home-made bowl of spaghetti and a superlative haute cuisine dish made from the same recipe depend not only on the ingredients they contain, but also on the way and order they are put together. Similarly, an experienced specialist will finely tune drug choices differently from a general practitioner attempting to comply with the same treatment guidelines.

The KoGNET study holds several messages: it clearly supports disease remission as the dominant determinant of 


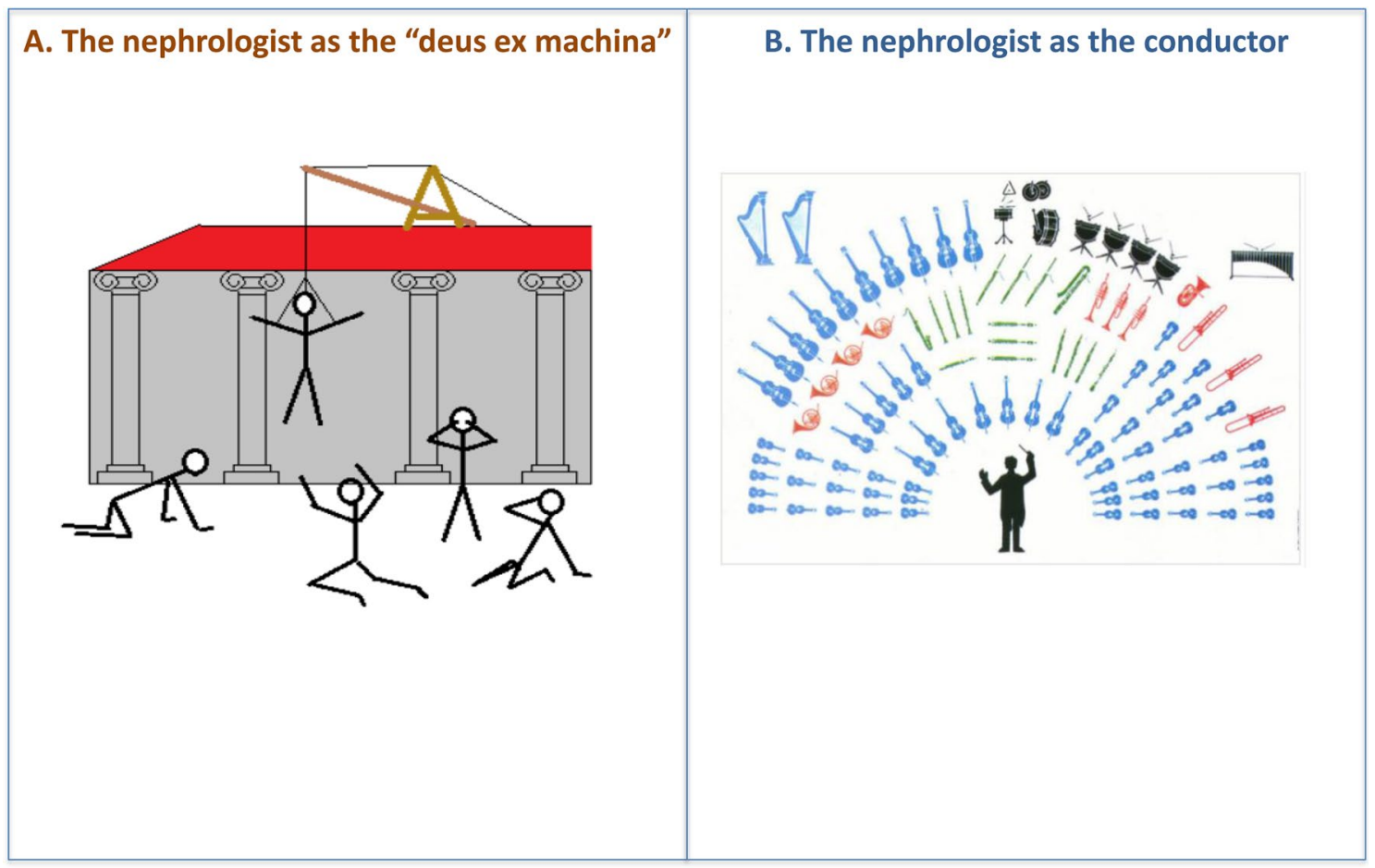

Fig. 1 Two "extreme" models of care: the nephrologist as deus ex machina and the nephrologist as the conductor of care. a The key choices are determined by the specialist, whose intervention is punctual and resolutive. Routine care becomes the responsibility of other professional figures that follow the expert's advice. In this model, decisions regarding hypertension treatment, lifestyle interventions, and follow-up are up to other partners on the care team. b Once the

renal survival in patients with glomerulonephritis. However, the paper sees blood-pressure control as a novel independent add-on factor in renal survival. This finding should compel nephrologists to carefully scrutinize whether this apparently trivial aspect of care could be improved in glomerulonephritis patients. Seen from this perspective, nothing is unimportant in glomerulonephritis treatment, and all aspects must be carefully monitored, since optimization of mundane details affects the renal prognosis.

At present many large nephrology centers favor prescription of complex therapies, leaving the optimization of routine issues such as hypertension, life-style or diet to other professionals. Given the KoGNET data, rethinking global nephrology patient care appears to be imperative if we wish to ensure optimal management of glomerular diseases [8] (Fig. 1).

\section{Compliance with ethical standards}

Conflict of interest The authors declare they have no conflict of interest. key choices have been made by the specialist, whose role includes coordinating care, other team members are advised and treatment begins. Routine care may engage other professional figures working under the expert's supervision. In this model, hypertension treatment, lifestyle interventions and follow-up are coordinated and overseen by the nephrologist

Ethical approval This article does not contain any studies with human participants performed by any of the authors.

\section{References}

1. Kim HW, Park JT, Su Joo Y, Kang SC, Lee JY, Lee S, Chang TI, Kang EW, Ryu DR, Yoo TH, Chin HJ, Kang SW, Han SH. Systolic Blood Pressure and Chronic Kidney Disease Progression in Patients with Primary Glomerular Disease. J Nephrol. https:// doi.org/10.1007/s40620-020-00930-x

2. Ku E, Lee BJ, Wei J, Weir MR (2019) Hypertension in CKD: core curriculum 2019. Am J Kidney Dis 74(1):120-131

3. Evangelidis N, Craig J, Bauman A, Manera K, Saglimbene V, Tong A (2019) Lifestyle behaviour change for preventing the progression of chronic kidney disease: a systematic review. BMJ Open 9(10):e031625

4. Georgianos PI, Agarwal R (2020) Hypertension in chronic kidney disease (CKD): diagnosis, classification and therapeutic targets. Am J Hypertens. hpaa209. [Online ahead of print]

5. Gaede P, Vedel P, Larsen N, Jensen GV, Parving HH, Pedersen O (2003) Multifactorial intervention and cardiovascular disease in patients with type 2 diabetes. N Engl J Med 348(5):383-393

6. Fishbane S, Agoritsas S, Bellucci A, Halinski C, Shah HH, Sakhiya V, Balsam L (2017) Augmented nurse care management in CKD stages 4 to 5: a randomized trial. Am J Kidney Dis 70(4):498-505 
7. Saudan P, Ponte B, Maragnon N, Martinez C, Berchtold L, Jaques D, Ernandez T, de Seigneux S, Carballo S, Perenger T, Martin PY (2020) Impact of superimposed nephrological care to guidelinesdirected management by primary care physicians of patients with stable chronic kidney disease: a randomized controlled trial. BMC Nephrol 21(1):128

8. Piccoli GB (2020) Who should be responsible for the care of advanced chronic kidney disease? Do the guidelines point to the end of nephrology follow-up of advanced CKD or are they the starting point for a new approach? BMC Nephrol 21:309

Publisher's Note Springer Nature remains neutral with regard to jurisdictional claims in published maps and institutional affiliations. 